6-30-2021

\title{
Pareto Distribution under Hybrid Censoring: Some Estimation
}

Gyan Prakash

Moti Lal Nehru Medical College, Allahabad, India, ggyanji@yahoo.com

Follow this and additional works at: https://digitalcommons.wayne.edu/jmasm

Part of the Applied Statistics Commons, Social and Behavioral Sciences Commons, and the Statistical Theory Commons

\section{Recommended Citation}

Prakash, Gyan (2021) "Pareto Distribution under Hybrid Censoring: Some Estimation," Journal of Modern Applied Statistical Methods: Vol. 19 : Iss. 1 , Article 16.

DOI: $10.22237 / j m a s m / 1619481660$

Available at: https://digitalcommons.wayne.edu/jmasm/vol19/iss1/16

This Regular Article is brought to you for free and open access by the Open Access Journals at DigitalCommons@WayneState. It has been accepted for inclusion in Journal of Modern Applied Statistical Methods by an authorized editor of DigitalCommons@WayneState. 


\section{Pareto Distribution under Hybrid Censoring: Some Estimation}

\section{Gyan Prakash}

Moti Lal Nehru Medical College

Allahabad, India

In the present study, the Pareto model is considered as the model from which observations are to be estimated using a Bayesian approach. Properties of the Bayes estimators for the unknown parameters have studied by using different asymmetric loss functions on hybrid censoring pattern and their risks have compared. The properties of maximum likelihood estimation and approximate confidence length have also been investigated under hybrid censoring. The performances of the procedures are illustrated based on simulated data obtained under the Metropolis-Hastings algorithm and a real data set.

Keywords: Hybrid censoring, approximate confidence length (ACL), invariant LINEX loss function (ILLF), generalized entropy loss function (GELF)

\section{Introduction}

The Pareto Type-II distribution is used here for Bayesian inference, having the probability density function

$$
\mathrm{f}(x ; \sigma, \theta)=\theta \sigma^{\theta}(x+\sigma)^{-(\theta+1)} ; \quad x \geq 0, \theta>0, \sigma>0 .
$$

Parameter $\theta$ is known as the shape parameter, whereas the parameter $\sigma$ is the scale parameter. The given model in Equation (1) is also called the Lomax distribution and is the result of a mixture of the Exponential distribution with scale parameter $\alpha$ and given scale parameter $\alpha$ is distributed as the Gamma density with parameters $\theta$ and $\sigma$.

The Pareto distribution provides a very flexible family of fat-tailed distributions and is a useful model for the income distribution of the higher income group. The model given in Equation (1) plays a vital role in socioeconomic studies

doi: 10.22237/jmasm/1619481660 | Accepted: Dec. 27, 2018; Published: June 30, 2021.

Correspondence: Gyan Prakash,ggyanji@yahoo.com 


\section{GYAN PRAKASH}

also. It is frequently used as a model for examining areas including city population distribution, stock price variation, oil field sites, and armed areas. It is suitable for approximating the right tails of distributions with positive skewness. The Pareto distribution has a decreasing failure rate, so it has repeatedly been used to model survival after some medical procedures (the ability to survive for a long time appears to increase the longer one survives after certain medical procedures).

The Pareto distribution plays an important role in a variety of other problems and was discussed by Steindle (1965) for size of cities and firms, business mortality by Lomax (1954), and service time in the queuing system by Harris (1967). A lot of work is available on the Pareto model, and very few of them are stated here. AlHussaini et al. (2001) obtained the Bayes prediction bounds for Type-I censored data from a finite mixture of Lomax components. Madi and Raqab (2004) used the Pareto model in the forecasting of temperature records.

D. C. Singh et al. (2007) assumed a classical Pareto model for the testimation of unknown parameters under the Linex loss function. Li (2011) discussed the maximum likelihood and Bayes estimates for reliability parameters of the Pareto model by using progressive Type-II censored samples. Al-Zahrani and Al-Sobhi (2013) used Lomax distribution based on general progressive censored data for evaluating the problem of the probability $S=\mathrm{P}(Y<X)$. The Bayes prediction bound lengths for the Pareto Type-II model were obtained by Prakash and Singh (2013) by using several different censoring criteria.

Okasha (2014), in his article about the E-Bayesian method, considered computing estimates of the unknown parameter, reliability, and hazard functions of Lomax distribution based on Type-II censored data. Prakash (2014) inspecting the Bayes estimators under right ordered sample data for the Lomax model. Some statistical inference for the two-parameter Pareto distribution based on Progressive Type-II censored data were discussed recently by Prakash (2017).

The objective of the present study is to investigate Bayesian inferences for unknown parameters of the underlying distribution based on hybrid censored data. The Bayes estimators under two different asymmetric loss functions, maximum likelihood estimators and ACL, have been obtained. The risks of the Bayes estimators have been compared within different asymmetric loss functions. The performances of the procedures are illustrated by a simulation technique based on the Metropolis-Hastings (M-H) algorithm and by a real data example was discussed by Lawless (1982). 


\section{PARETO DISTRIBUTION UNDER HYBRID CENSORING}

\section{Hybrid Censoring \& ML Estimation}

The conventional Type-I and Type-II censoring schemes are the most popular censoring schemes for reliability analysis. The considered hybrid censoring scheme is a mixture of Type-I and Type-II censoring schemes. Some recent studies on hybrid censoring have been carried out by many authors, including Childs et al. (2003), Kundu (2007), Banerjee and Kundu (2008), Dube et al. (2011), Balakrishnan and Kundu (2013), B. Singh et al. (2014), and Kayal et al. (2017).

Let us assume a total of $n$ identical units are put on a test. Under the hybrid censoring scheme, the test is terminated when a pre-assigned number $m$ (say) out of $n$ units have failed or when a pre-determined time $t$ has been reached. Hence, in the hybrid censoring scheme, $m$ and $t$ are respectively assumed as the number of failures and the experimental time. The test will not exceed $m$ or $t$, respectively.

Now let us assume the lifespans of the test units are $T_{1}, T_{2}, \ldots, T_{n}$ and are identically independently distributed with density function Equation (1). Let $T_{1: n} \leq T_{2: n} \leq \ldots \leq T_{n: n}$ be the corresponding order statistic. Let the number of failures and the observation times be denoted by $M$ and $T=\min \left(T_{m: n}, t\right)$, respectively. Therefore, the observed samples are assumed to be $\left(T_{1: n}, T_{2: n}, \ldots \leq T_{M: n} ; M\right)$.

The likelihood function based on hybrid censored data is defined when $t_{0}$ and $d$ are the observed values of $T$ and $M$, respectively, as

$$
\begin{aligned}
& \mathrm{L}(\underline{x} \mid \sigma, \theta) \propto \prod_{i=1}^{d} \mathrm{f}\left(x_{(i)} ; \sigma, \theta\right)\left(1-\mathrm{F}\left(t_{(0)} ; \sigma, \theta\right)\right)^{n-d} \\
& \propto \prod_{i=1}^{d}\left(\theta \sigma^{\theta}\left(x_{(i)}+\sigma\right)^{-(\theta+1)}\right)\left(\sigma^{\theta}\left(t_{(0)}+\sigma\right)^{-\theta}\right)^{n-d} \\
& \mathrm{~L}(\underline{x} \mid \sigma, \theta) \propto \theta^{d} \sigma^{n \theta} \exp \left(-\theta H_{1}-H_{0}\right)
\end{aligned}
$$

where

$$
H_{0}=\sum_{i=1}^{d} \log \left(x_{(i)}+\sigma\right) \text { and } H_{1}=\sum_{i=1}^{d} \log \left(x_{(i)}+\sigma\right)+(n-d) \log \left(t_{(0)}+\sigma\right) \text {. }
$$

Taking the logarithm on Equation (2) and differentiating with respect to the unknown parameters, we get 


\section{GYAN PRAKASH}

$$
\begin{aligned}
& \frac{\partial}{\partial \theta} \log \mathrm{L}(\underline{x} \mid \sigma, \theta)=\frac{d}{\theta}+n \log \sigma-H_{1} \\
& \frac{\partial}{\partial \sigma} \log \mathrm{L}(\underline{x} \mid \sigma, \theta)=\frac{n \theta}{\sigma}-\theta\left\{\sum_{i=1}^{d}\left(\frac{1}{x_{(i)}+\sigma}\right)+\frac{n-d}{t_{(0)}+\sigma}\right\}-\sum_{i=1}^{d}\left(\frac{1}{x_{(i)}+\sigma}\right)
\end{aligned}
$$

Hence, the maximum likelihood estimators corresponding to the parameters $\theta$ and $\sigma$ are denoted by $\hat{\theta}_{\mathrm{Ml}}$ and $\hat{\sigma}_{\mathrm{Ml}}$, respectively, and obtained as

$$
\begin{gathered}
\hat{\theta}_{\mathrm{Ml}}=\frac{d}{H_{1}-n \log \sigma} \\
\hat{\sigma}_{\mathrm{Ml}}=\frac{n \hat{\theta}_{\mathrm{Ml}}}{\hat{\theta}_{\mathrm{Ml}}\left\{\sum_{i=1}^{d}\left(\frac{1}{x_{(i)}+\sigma}\right)+\frac{n-d}{t_{(0)}+\sigma}\right\}+\sum_{i=1}^{d}\left(\frac{1}{x_{(i)}+\sigma}\right)}=\mathrm{h}(\sigma), \text { say. }
\end{gathered}
$$

A simple iterative procedure was adopted for solving Equation (4). For this, let $\sigma^{(0)}$ be an initial guess value of $\sigma$. Then successive approximations of $\sigma$ are $\sigma^{(1)}=\mathrm{h}\left(\sigma^{(0)}\right)$, $\sigma^{(2)}=\mathrm{h}\left(\sigma^{(1)}\right), \ldots, \sigma^{(m+1)}=\mathrm{h}\left(\sigma^{(m)}\right)$. Stop the iterative procedure at the $m^{\text {th }}$ stage if $\left|\sigma^{(m+1)}-\sigma^{(m)}\right|<\varphi$ for some pre-specified small value $\varphi$.

Now, the second derivatives of the logarithm of the likelihood function are obtained and given as

$$
\begin{gathered}
\frac{\partial^{2}}{\partial \theta^{2}} \log \mathrm{L}(\underline{x} \mid \sigma, \theta)=-\frac{d}{\theta^{2}} \\
\frac{\partial^{2}}{\partial \sigma^{2}} \log \mathrm{L}(\underline{x} \mid \sigma, \theta)=-\frac{n \theta}{\sigma^{2}}+\theta\left\{\sum_{i=1}^{d} \frac{1}{\left(x_{(i)}+\sigma\right)^{2}}+\frac{n-d}{\left(t_{(0)}+\sigma\right)^{2}}\right\}+\sum_{i=1}^{d} \frac{1}{\left(x_{(i)}+\sigma\right)^{2}},
\end{gathered}
$$

and

$$
\frac{\partial^{2}}{\partial \theta \partial \sigma} \log \mathrm{L}(\underline{x} \mid \sigma, \theta)=\frac{n}{\sigma}-\left\{\sum_{i=1}^{d} \frac{1}{x_{(i)}+\sigma}+\frac{n-d}{t_{(0)}+\sigma}\right\}=\frac{\partial^{2}}{\partial \sigma \partial \theta} \log \mathrm{L}(\underline{x} \mid \sigma, \theta) .
$$




\section{PARETO DISTRIBUTION UNDER HYBRID CENSORING}

The observed information matrix is denoted by $\mathbf{I}(\boldsymbol{\Theta})$ and defined, for $\hat{\boldsymbol{\Theta}}=(\hat{\theta}, \hat{\sigma})$ the ML estimation of the parameter $\boldsymbol{\Theta}=(\theta, \sigma)$, as

$$
\mathbf{I}(\boldsymbol{\Theta})=\left[\begin{array}{cc}
-\frac{\partial^{2}}{\partial \theta^{2}} \log \mathrm{L}(\underline{x} \mid \sigma, \theta) & -\frac{\partial^{2}}{\partial \theta \partial \sigma} \log \mathrm{L}(\underline{x} \mid \sigma, \theta) \\
-\frac{\partial^{2}}{\partial \sigma \partial \theta} \log \mathrm{L}(\underline{x} \mid \sigma, \theta) & -\frac{\partial^{2}}{\partial \sigma^{2}} \log \mathrm{L}(\underline{x} \mid \sigma, \theta)
\end{array}\right]_{(\hat{\theta}, \hat{\sigma})}
$$

Following Park and Balakrishnan (2009), the Fisher Information matrix is obtained by

$$
\ell(\boldsymbol{\Theta})=\left[\begin{array}{ll}
\ell_{11}(\boldsymbol{\Theta}) & \ell_{12}(\boldsymbol{\Theta}) \\
\ell_{21}(\boldsymbol{\Theta}) & \ell_{22}(\boldsymbol{\Theta})
\end{array}\right]
$$

where

$$
\begin{aligned}
& \ell_{11}(\boldsymbol{\Theta})=\int_{0}^{t}\left\{\frac{\partial}{\partial \theta} \log \rho(x ; \boldsymbol{\Theta})\right\}^{2} \sum_{i=1}^{m} \mathrm{f}_{i: n}(x ; \boldsymbol{\Theta}) d x \\
& \ell_{22}(\boldsymbol{\Theta})=\int_{0}^{t}\left\{\frac{\partial}{\partial \sigma} \log \rho(x ; \boldsymbol{\Theta})\right\}^{2} \sum_{i=1}^{m} \mathrm{f}_{i: n}(x ; \boldsymbol{\Theta}) d x \\
& \ell_{12}(\boldsymbol{\Theta})=\ell_{21}(\boldsymbol{\Theta})=\int_{0}^{t}\left\{\frac{\partial}{\partial \theta} \log \rho(x ; \boldsymbol{\Theta})\right\}\left\{\frac{\partial}{\partial \sigma} \log \rho(x ; \boldsymbol{\Theta})\right\} \sum_{i=1}^{m} \mathrm{f}_{i: n}(x ; \boldsymbol{\Theta}) d x
\end{aligned}
$$

Here, $\rho(x ; \boldsymbol{\Theta})$ and $\mathrm{f}_{i: n}(x ; \boldsymbol{\Theta})$ are known as the hazard function for Equation (1) and the probability density of $X_{i: n}$, respectively, and defined as

$$
\rho(x ; \boldsymbol{\Theta})=\frac{\theta}{x+\sigma}
$$

and

$$
\mathrm{f}_{i: n}(x ; \boldsymbol{\Theta})=i\left(\begin{array}{l}
n \\
i
\end{array}\right)(\mathrm{F}(x ; \sigma, \theta))^{i-1}(1-\mathrm{F}(x ; \sigma, \theta))^{n-i} \mathrm{f}(x ; \sigma, \theta), \quad i=1,2, \ldots, m
$$




\section{GYAN PRAKASH}

$$
\Rightarrow \mathrm{f}_{i: n}(x ; \boldsymbol{\Theta})=i\left(\begin{array}{l}
n \\
i
\end{array}\right)\left(1-\left(\frac{\sigma}{x+\sigma}\right)^{\theta}\right)^{i-1} \frac{\theta \sigma^{\theta(n-i+1)}}{(x+\sigma)^{\theta(n-i+1)+1}}
$$

Hence,

$$
\begin{aligned}
& \ell_{11}(\boldsymbol{\Theta})=\sum_{i=1}^{m} i\left(\begin{array}{l}
n \\
i
\end{array}\right) \int_{0}^{t}\left(1-\left(\frac{\sigma}{x+\sigma}\right)^{\theta}\right)^{i-1} \frac{\sigma^{\theta(n-i+1)}}{\theta(x+\sigma)^{\theta(n-i+1)+1}} d x \\
& \ell_{22}(\boldsymbol{\Theta})=\sum_{i=1}^{m} i\left(\begin{array}{l}
n \\
i
\end{array}\right) \int_{0}^{t}\left(1-\left(\frac{\sigma}{x+\sigma}\right)^{\theta}\right)^{i-1} \frac{\theta \sigma^{\theta(n-i+1)}}{(x+\sigma)^{\theta(n-i+1)+3}} d x
\end{aligned}
$$

and

$$
\ell_{12}(\boldsymbol{\Theta})=\ell_{21}(\boldsymbol{\Theta})=\sum_{i=1}^{m} i\left(\begin{array}{c}
n \\
i
\end{array}\right) \int_{0}^{t}\left(1-\left(\frac{\sigma}{x+\sigma}\right)^{\theta}\right)^{i-1} \frac{\sigma^{\theta(n-i+1)}}{(x+\sigma)^{\theta(n-i+1)+2}} d x
$$

It is clear that the elements of $\ell(\boldsymbol{\Theta})$ from Equation (6) are to be computed numerically.

\section{The Bayes Estimation under Asymmetric Loss Function}

Prakash (2017) defined the joint prior density function, when both the parameter of the underlying distribution given in Equation (1) are assumed as the random variable, and given as

$$
\pi(\theta, \sigma)=\frac{\gamma^{\beta}}{\Gamma(\beta) \Gamma(\alpha)} \theta^{\alpha-1} e^{-\alpha \theta} \sigma^{\alpha+\beta-1} e^{-\gamma \sigma}
$$

The joint posterior and marginal posterior densities corresponding to the parameters $\theta$ and $\sigma$ are obtained as

$$
\pi_{(\theta, \sigma)}^{*}=\Omega \theta^{\alpha+d-1} \sigma^{\alpha+\beta-1} e^{-\gamma \sigma} e^{-H_{0}} e^{-\theta\left(\sigma+H_{1}-n \log \sigma\right)},
$$




\section{PARETO DISTRIBUTION UNDER HYBRID CENSORING}

$$
\pi_{\theta}^{*}=\Omega \theta^{\alpha+d-1} \int_{\sigma} \sigma^{\alpha+\beta-1} e^{-\gamma \sigma} e^{-H_{0}} e^{-\theta\left(\sigma+H_{1}-n \log \sigma\right)} d \sigma
$$

and

$$
\pi_{\sigma}^{*}=\Omega \frac{\Gamma(\alpha+d)}{\left(\sigma+H_{1}-n \log \sigma\right)^{\alpha+d}} \sigma^{\alpha+\beta-1} e^{-\gamma \sigma} e^{-H_{0}},
$$

where

$$
\Omega=\left\{\Gamma(\alpha+d) \int_{\sigma} \frac{e^{-\gamma \sigma} e^{-H_{0}}}{\left(\sigma+H_{1}-n \log \sigma\right)^{\alpha+d}} \sigma^{\alpha+\beta-1} d \sigma\right\}^{-1} .
$$

The greatest number of Bayesian inference was developed under the usual symmetric loss function called the squared error loss function. The squared error loss is symmetrical and gives equal importance to the losses due to overestimation and underestimation. Parsian and Kirmani (2002) discussed the infeasibility of the squared error loss in most practical situations. Based on the practical importance of the Pareto distribution, a useful and flexible class of asymmetric loss functions, called Invariant LINEX Loss Functions (ILLFs) has used for the Bayesian inference. Following Prakash (2015) the ILLF is defined for an estimate $\hat{\boldsymbol{\Theta}}$ of the parameter $\boldsymbol{\Theta}$ as

$$
\mathrm{L}_{\mathrm{I}}(\partial)=e^{c \partial}-c \partial-1 ; \quad c \neq 0, \partial=\boldsymbol{\Phi}-1, \boldsymbol{\Phi}=\frac{\hat{\boldsymbol{\Theta}}}{\boldsymbol{\Theta}}
$$

The parameter $c$ is defined as the shape parameter of the ILLF. See Prakash (2015) for more details regarding the ILLF. The Bayes estimators for the parameters $\theta$ and $\sigma$ under ILLF are obtained by solving following equality for each parameter:

$$
\int_{\Theta}\left\{\frac{1}{\Theta} \exp \left(-c \frac{\hat{\Theta}_{\mathrm{L}}}{\Theta}\right)\right\} \pi_{\Theta}^{*} d \Theta=e^{c} \int_{\Theta} \frac{1}{\Theta} \pi_{\Theta}^{*} d \Theta ; \quad \Theta=\theta, \sigma .
$$

Some numerical methods are applied here for the analysis of the proposed methods due to the non-existence of a closed expressions for the Bayes estimators. 


\section{GYAN PRAKASH}

Several authors have already explored in their articles that the Bayes estimators under the LINEX loss function perform better than the squared error loss function. We are not going for the same. Here, we considered another asymmetric loss function named the Generalized Entropy Loss Function (GELF) and study the performances of the Bayes estimators for a selected set of parametric values.

The ILLF grows almost exponentially on one side of zero and nearly linearly on the other side. A suitable alternative of ILLF is a GELF, which is defined for any estimate $\hat{\Theta}$ as

$$
\mathrm{L}_{\mathrm{E}}(\Phi)=(\Phi)^{b}-b \log (\Phi)-1 ; \quad b \neq 0
$$

The parameter $b$ is the shape parameter of the GELF. See Calabria and Pulcini (1996) for more detail about the entropy loss function and P. K. Singh et al. (2008) for GELF.

Now, the Bayes estimators corresponding to the parameters $\theta$ and $\sigma$ under GELF are obtained by solving following the equality for each parameter:

$$
\hat{\Theta}_{\mathrm{E}}=\left\{\int_{\Theta} \Theta^{-d} \pi_{\Theta}^{*} d \Theta\right\}^{-\frac{1}{d}} ; \quad \Theta=\theta, \sigma .
$$

\section{Metropolis-Hastings Algorithm}

Metropolis et al. (1953) and Hastings (1970) have discussed an algorithm called the Metropolis-Hastings (M-H) algorithm, which is widely used to simulate samples from a given posterior distribution by making use of an arbitrary proposal distribution and provides an alternative way for computing Bayes estimates.

The sample was generated from the posterior distribution given in Equation (12) in following way:

Let us assume that parameters $\theta$ and $\sigma$ are independently distributed as normal. In order to simulate replicates from the prescribed distribution, we need to implement following steps:

1:- $\quad$ Select an initial guess of $\delta=(\theta, \sigma)$ and set it as $\delta_{0}=\left(\theta_{0}, \sigma_{0}\right)$.

2:- Now, generate a new $\delta^{\prime}$ by using the proposed $\mathrm{N}\left(\delta_{n-1}, \boldsymbol{\Sigma}\right)$ distribution. Here $n$ is the iterative stage and $\boldsymbol{\Sigma}$ is the variance-covariance matrix. 


\section{PARETO DISTRIBUTION UNDER HYBRID CENSORING}

3:- $\quad$ Now, compute $h=\min \left\{1, \pi_{\left(\delta^{\prime}\right)}^{*} / \pi_{\left(\delta_{n-1}^{\prime}\right)}^{*}\right\}$.

4:- $\quad$ Then generate a sample $u$ from the Uniform distribution $\mathrm{U}(0,1)$.

5:- $\quad$ If $u \leq h$, then set $\delta_{n} \rightarrow \delta^{\prime}$

Otherwise $\delta_{n} \rightarrow \delta_{n-1}$.

6:- Repeat steps 2-5 up to $N$ times and collect an adequate number of replicates. Then estimate the associated Bayes estimates and the corresponding risks of the parameters under for the concerned loss functions.

In the previous sections, we proposed different estimators for the unknown parameters of the underlying distribution. Now we assess their behavior in terms of risk by using hybrid censored samples. These values are computed using Monte Carlo simulations on the basis of 10,000 replications.

The performance of the ML estimators under squared error loss function and the approximate confidence lengths are presented in Table 1 and Table 2 based on a simulation study under the hybrid censoring pattern. Prakash (2014) studied the Bayes estimators of the concerned distribution under the right censored data and noted that the risks were minimized for smaller $\sigma$ (small values of scale parameter). Thus, in the present study, the pre assumed values of $\sigma$ are $0.25,0.50$, and 1.00 and the assumed values of shape parameter are $\theta=0.50,1.00,2.00$, and 5.00.

Table 1. Risk of maximum likelihood estimators

\begin{tabular}{|c|c|c|c|c|c|c|c|c|}
\hline \multirow{2}{*}{$\theta=2.00$} & \multirow[b]{2}{*}{$m$} & & \multicolumn{3}{|c|}{$\begin{array}{l}\text { Based on simulated data } \\
\qquad \sigma\end{array}$} & \multicolumn{3}{|c|}{$\begin{array}{l}\text { Based on real data } \\
\sigma \sigma\end{array}$} \\
\hline & & $t$ & 0.25 & 0.50 & 1.00 & 0.25 & 0.50 & 1.00 \\
\hline \multirow[t]{6}{*}{$\hat{\theta}_{\mathrm{MI}}$} & 5 & 1.00 & 0.6481 & 0.6633 & 0.6716 & 0.5818 & 0.6012 & 0.6039 \\
\hline & 10 & & 0.6325 & 0.6505 & 0.6563 & 0.5524 & 0.5842 & 0.5883 \\
\hline & 15 & & 0.6053 & 0.6219 & 0.6475 & 0.5335 & 0.5487 & 0.5571 \\
\hline & 5 & 5.00 & 0.7269 & 0.7476 & 0.7545 & 0.6523 & 0.6564 & 0.6771 \\
\hline & 10 & & 0.6974 & 0.7223 & 0.7237 & 0.6077 & 0.6491 & 0.6491 \\
\hline & 15 & & 0.6702 & 0.6937 & 0.7101 & 0.5901 & 0.6122 & 0.6371 \\
\hline \multirow[t]{6}{*}{$\hat{\sigma}_{\mathrm{MI}}$} & 5 & 1.00 & 0.9148 & 0.9358 & 0.9473 & 0.8233 & 0.8501 & 0.8538 \\
\hline & 10 & & 0.8266 & 0.8514 & 0.8594 & 0.7161 & 0.7399 & 0.7455 \\
\hline & 15 & & 0.8074 & 0.8303 & 0.8657 & 0.7082 & 0.7292 & 0.7608 \\
\hline & 5 & 5.00 & 1.0237 & 1.0522 & 1.0618 & 0.9206 & 0.9263 & 0.9549 \\
\hline & 10 & & 0.9162 & 0.9506 & 0.9525 & 0.7923 & 0.8495 & 0.8495 \\
\hline & 15 & & 0.8971 & 0.9295 & 0.9541 & 0.7864 & 0.8169 & 0.8513 \\
\hline
\end{tabular}




\section{GYAN PRAKASH}

The simulation is carried out for a particular set of hybrid censored data of size $n(=20)$, and different choices of $m$, $t$, and level of significance $\varepsilon$. It is observed that the risk magnitude decreases when the censored sample size $m$ increases, whereas the opposite trend is seen when the pre-determined failure time $t$ increases.

Similar properties also are seen when the scale parameter $\sigma$ increases. However, in the case of $\hat{\sigma}_{\mathrm{Ml}}$ it is noted that the risk magnitude first increases up to $\sigma \leq 0.50$ and then decreases. The effect of variation in the shape parameter $\theta$ is also observed. A minor reduction in the magnitude of the Bayes risk has been noted when the values of the shape parameter increase. The minimum risk magnitude was observed for $\theta=2.00$. Hence, all the results discussed here are only for $\theta=2.00$.

Table 2. Asymptotic confidence interval

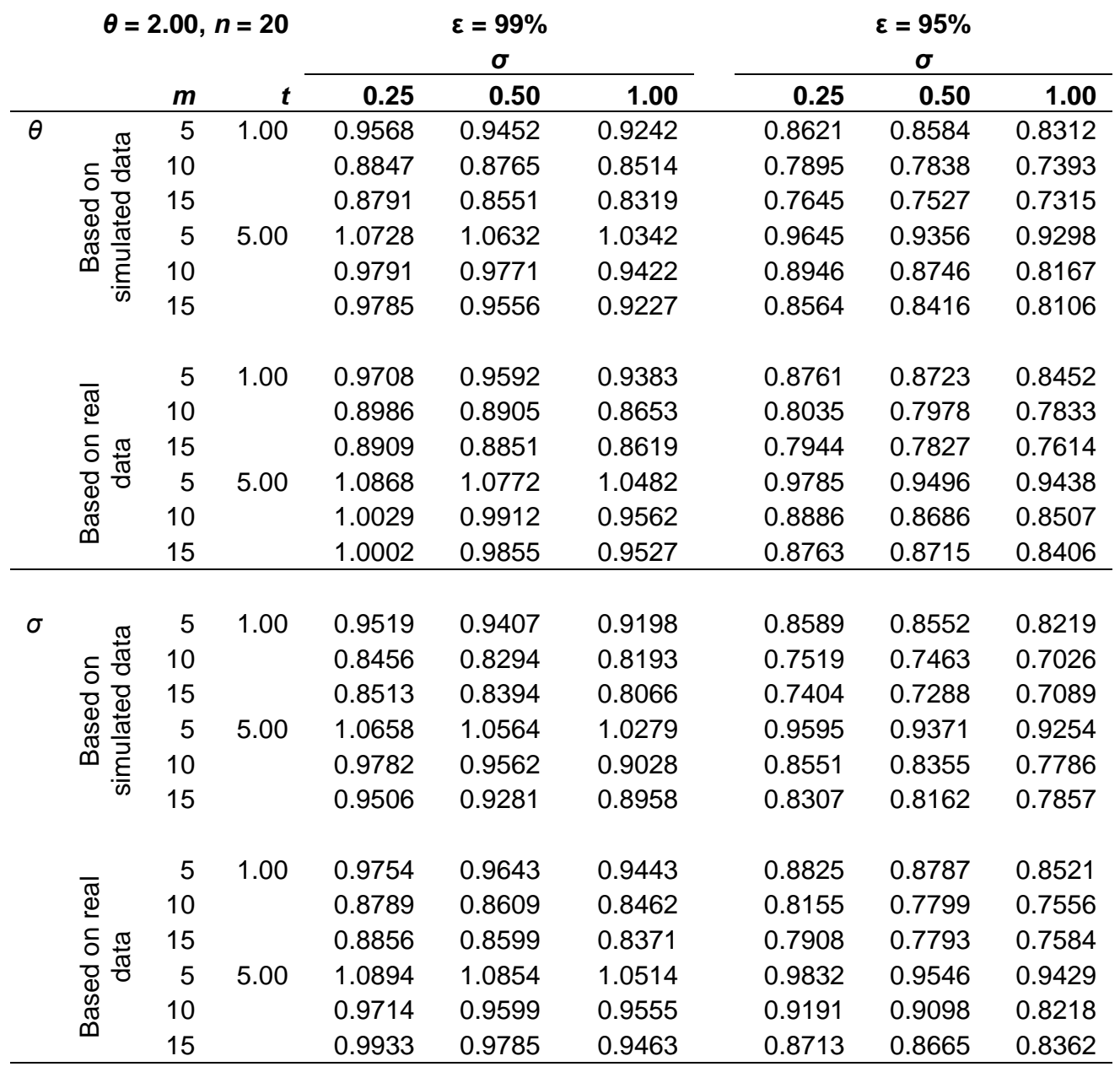




\section{PARETO DISTRIBUTION UNDER HYBRID CENSORING}

Table 3. Risk magnitude under the ILLF

\begin{tabular}{|c|c|c|c|c|c|c|c|c|c|}
\hline & \multicolumn{3}{|c|}{$\theta=2.00, n=20$} & \multicolumn{3}{|c|}{$\begin{array}{l}\text { Based on simulated data } \\
\qquad \sigma \\
\end{array}$} & \multicolumn{3}{|c|}{$\begin{array}{c}\text { Based on real data } \\
\qquad \sigma \\
\end{array}$} \\
\hline & $c$ & $m$ & $t$ & 0.25 & 0.50 & 1.00 & 0.25 & 0.50 & 1.00 \\
\hline \multirow[t]{18}{*}{$\hat{\theta}_{\mathrm{L}}$} & 0.25 & 5 & 1.00 & 0.6963 & 0.7122 & 0.7207 & 0.6274 & 0.6476 & 0.6504 \\
\hline & & 10 & & 0.6041 & 0.6213 & 0.6479 & 0.5295 & 0.5453 & 0.5542 \\
\hline & & 15 & & 0.5861 & 0.6047 & 0.6107 & 0.5029 & 0.5359 & 0.5401 \\
\hline & & 5 & 5.00 & 0.7781 & 0.7996 & 0.8067 & 0.7006 & 0.7049 & 0.7264 \\
\hline & & 10 & & 0.6714 & 0.6958 & 0.7129 & 0.5883 & 0.6112 & 0.6371 \\
\hline & & 15 & & 0.6534 & 0.6792 & 0.6807 & 0.5603 & 0.6032 & 0.6032 \\
\hline & 0.50 & 5 & 1.00 & 0.6619 & 0.6772 & 0.6855 & 0.5954 & 0.6148 & 0.6175 \\
\hline & & 10 & & 0.6008 & 0.6188 & 0.6382 & 0.5238 & 0.5523 & 0.5564 \\
\hline & & 15 & & 0.5959 & 0.6125 & 0.6247 & 0.5204 & 0.5393 & 0.5475 \\
\hline & & 5 & 5.00 & 0.7415 & 0.7618 & 0.7687 & 0.6661 & 0.6702 & 0.6913 \\
\hline & & 10 & & 0.6659 & 0.6909 & 0.7011 & 0.5806 & 0.6174 & 0.6278 \\
\hline & & 15 & & 0.6612 & 0.6846 & 0.6923 & 0.5759 & 0.6028 & 0.6174 \\
\hline & 1.00 & 5 & 1.00 & 0.9142 & 0.9353 & 0.9467 & 0.8222 & 0.8491 & 0.8528 \\
\hline & & 10 & & 0.8297 & 0.8546 & 0.8814 & 0.7233 & 0.7628 & 0.7684 \\
\hline & & 15 & & 0.8229 & 0.8459 & 0.8627 & 0.7187 & 0.7444 & 0.7561 \\
\hline & & 5 & 5.00 & 1.0234 & 1.0521 & 1.0616 & 0.9201 & 0.9256 & 0.9543 \\
\hline & & 10 & & 0.9197 & 0.9542 & 0.9682 & 0.8018 & 0.8527 & 0.8671 \\
\hline & & 15 & & 0.9129 & 0.9455 & 0.9561 & 0.7953 & 0.8325 & 0.8527 \\
\hline \multirow[t]{18}{*}{$\hat{\sigma}_{\mathrm{L}}$} & 0.25 & 5 & 1.00 & 0.7076 & 0.7233 & 0.7321 & 0.6387 & 0.6589 & 0.6617 \\
\hline & & 10 & & 0.6381 & 0.6553 & 0.6819 & 0.5635 & 0.5793 & 0.5882 \\
\hline & & 15 & & 0.5957 & 0.6144 & 0.6204 & 0.5126 & 0.5456 & 0.5498 \\
\hline & & 5 & 5.00 & 0.7894 & 0.8109 & 0.8182 & 0.7119 & 0.7162 & 0.7377 \\
\hline & & 10 & & 0.7054 & 0.7298 & 0.7469 & 0.6223 & 0.6452 & 0.6711 \\
\hline & & 15 & & 0.6631 & 0.6889 & 0.6904 & 0.5701 & 0.6129 & 0.6129 \\
\hline & 0.50 & 5 & 1.00 & 0.6732 & 0.6885 & 0.6968 & 0.6067 & 0.6261 & 0.6288 \\
\hline & & 10 & & 0.6348 & 0.6528 & 0.6722 & 0.5578 & 0.5863 & 0.5904 \\
\hline & & 15 & & 0.6056 & 0.6222 & 0.6344 & 0.5301 & 0.5487 & 0.5572 \\
\hline & & 5 & 5.00 & 0.7523 & 0.7731 & 0.7803 & 0.6774 & 0.6815 & 0.7023 \\
\hline & & 10 & & 0.6999 & 0.7249 & 0.7351 & 0.6146 & 0.6514 & 0.6618 \\
\hline & & 15 & & 0.6707 & 0.6943 & 0.7021 & 0.5856 & 0.6125 & 0.6271 \\
\hline & 1.00 & 5 & 1.00 & 0.9255 & 0.9466 & 0.9581 & 0.8335 & 0.8604 & 0.8641 \\
\hline & & 10 & & 0.8637 & 0.8886 & 0.9154 & 0.7573 & 0.7968 & 0.8024 \\
\hline & & 15 & & 0.8326 & 0.8556 & 0.8724 & 0.7284 & 0.7541 & 0.7658 \\
\hline & & 5 & 5.00 & 1.0347 & 1.0634 & 1.0729 & 0.9313 & 0.9369 & 0.9656 \\
\hline & & 10 & & 0.9537 & 0.9882 & 1.0022 & 0.8358 & 0.8867 & 0.9011 \\
\hline & & 15 & & 0.9226 & 0.9552 & 0.9658 & 0.8051 & 0.8422 & 0.8624 \\
\hline
\end{tabular}




\section{GYAN PRAKASH}

Table 4. Risk magnitude under the GELF

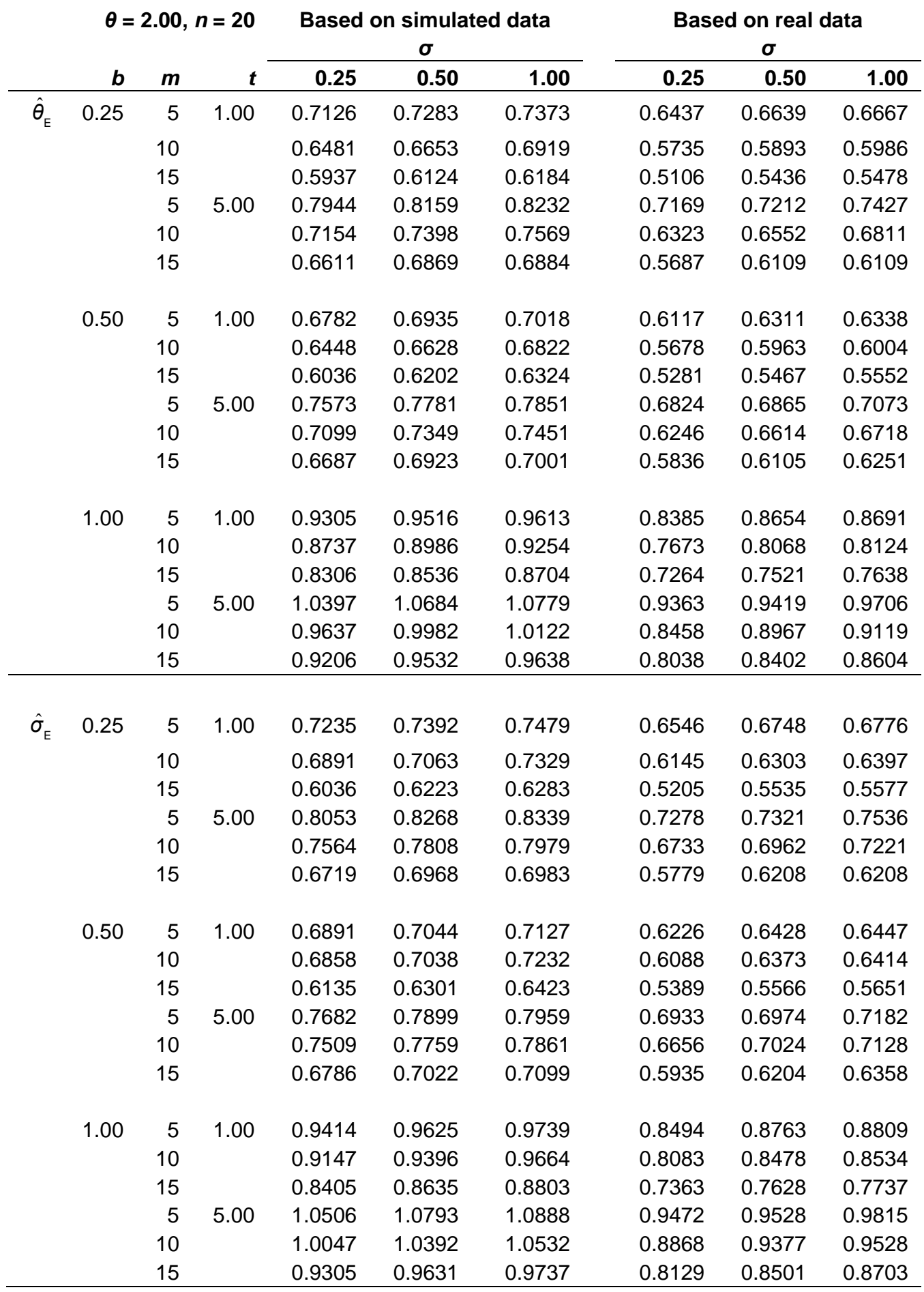




\section{PARETO DISTRIBUTION UNDER HYBRID CENSORING}

Table 2 presents the asymptotic confidence length (ACL) for selected parametric values and significance levels of $99 \%$ and $95 \%$. It is observed that the smaller censored sample size provides a wider ACL. Similar properties are also seen for $\sigma$. The larger significance level or the larger time span $t$ gives a wider length. That means, as the significance level or the time span becomes narrower, the ACL also becomes narrower.

We obtain the risks for the Bayes estimators based on the assumption that the unknown hyper parameters of the prior distribution assume arbitrary values as $\alpha=0.25, \beta=1.00$, and $\gamma=3.25$. The risks of the Bayes estimators are derived by using the M-H algorithm. Further, the shape parametric values of the loss functions are selected for prior study based on underlying distribution. Tables 3 and 4 presents the risks of the Bayes estimators under the ILLF and GELF, respectively.

For the risks for the Bayes estimators under ILLF, all the properties seen are similar to those discussed above for the ML estimator. Further, the risk magnitudes are found to be minimum for $c=0.50$. However, the tendency of the risk magnitude is to become smaller first and then wider, except for a small time span $t$ and large censored sample size $m$. Table 4 shows similar properties as discussed for ILLF for the risk obtained for Bayes estimators under GELF. It is further noted that the risk magnitude is smaller for ILLF as compared to GELF when other parametric values are fixed.

It is also noted that the risk magnitude under GELF for the Bayes estimators obtained under ILLF gives the higher risk magnitude when compared with the risk obtained under ILLF. Similarly, a trend is also seen for the Bayes estimator under GELF and risk obtained by using the invariant LINEX loss criterion. However, these tables are not included in the article.

\section{Numerical Illustration}

In the present section the properties of Bayesian inference are studied by real-life data. Lawless (1982) considered the data representing break-down times (in minutes) of an insulating fluid between electrodes at a voltage of $34 \mathrm{KV}$. A total of 18 observations are given in Table 5. Based on the above data, the numerical illustrations have been presented in Tables 1-4. All the properties are similar as discussed above for all the considered estimation criteria under the simulation. One remarkable point is that the risk magnitude is noted to be smaller when compared to the simulated data for all the considered fixed parametric values. Similarly, ACL is also found to be wider for the real data. 


\section{GYAN PRAKASH}

Table 5. The break-down times of an insulating fluid

$\begin{array}{rrrrrr}0.19 & 0.78 & 0.96 & 1.31 & 2.78 & 3.16 \\ 4.15 & 4.67 & 4.85 & 6.50 & 7.35 & 8.01 \\ 8.27 & 12.06 & 31.75 & 32.52 & 33.91 & 36.71\end{array}$

\section{Conclusion}

In the present article we discussed the Bayes risks under different asymmetric loss functions on the hybrid censoring pattern. The Pareto Type-II model is seen here in the Bayesian analysis for unknown parameters. The approximate confidence length and ML estimation are also presented. A simulation based on the MetropolisHastings algorithm was performed for illustrating the operations. The results are also verified with the help of a real data set. The risk magnitudes seen smaller for ILLF as compared to GELF when other parametric values are believed to be made. The performances of a real data set show better as compared to the simulated results.

\section{References}

Al-Hussaini, E. K., Jaheen, Z. F., \& Nigm, A. M. (2001). Bayesian prediction based on finite mixtures of Lomax components model and Type-I censoring. Statistics, 35(3), 259-268. doi: 10.1080/02331880108802735

Al-Zahrani, B., \& Al-Sobhi, M. (2013). On parameters estimation of Lomax distribution under general progressive censoring. Journal of Quality and Reliability Engineering, 2013, 431541. doi: 10.1155/2013/431541

Balakrishnan, N., \& Kundu, D. (2013). Hybrid censoring models, inferential results and applications. Computational Statistics \& Data Analysis, 57(1), 166209. doi: 10.1016/j.csda.2012.03.025

Banerjee, A., \& Kundu, D. (2008). Inference based on Type-II hybrid censored data from a Weibull distribution. IEEE Transactions on Reliability, 57(2), 369-378. doi: 10.1109/TR.2008.916890

Calabria, R., \& Pulcini, G. (1996). Point estimation under asymmetric loss functions for left-truncated exponential samples. Communication in Statistics Theory and Methods, 25(3), 585-600. doi: 10.1080/03610929608831715

Childs, A., Chandrasekhar, B., Balakrishnan, N., \& Kundu, D. (2003). Exact inference based on Type-I and Type-II hybrid censored samples from the exponential distribution. Annals of the Institute of Statistical Mathematics, 55(2), 319-330. doi: 10.1007/BF02530502 


\section{PARETO DISTRIBUTION UNDER HYBRID CENSORING}

Dube, S., Pradhan, B., \& Kundu, D. (2011). Parameter estimation of the hybrid censored log-normal distribution. Journal of Statistical Computation and Simulation, 81(3), 275-287. doi: 10.1080/00949650903292650

Harris, C. M. (1967). Queues with stochastic service rate. Naval Research Logistics Quarterly, 14(2), 219-230. 10.1002/nav.3800140207

Kayal, T., Tripathi, Y. M., Rastogi, M. K., \& Asgharzadeh, A. (2017). Inference for Burr XII distribution under Type-I progressive hybrid censoring. Communication in Statistics - Simulation and Computation, 46(9), 7447-7465. doi: 10.1080/03610918.2016.1241405

Kundu, D. (2007). On hybrid censored Weibull distribution. Journal of Statistical Planning and Inference, 137(7), 2127-2142. doi:

10.1016/j.jspi.2006.06.043

Lawless, J. F. (1982). Statistical models and methods for lifetime data. New York: Wiley.

Li, F. (2011). Reliability analysis for the Pareto model based on the progressive Type-II censored sample. In D. Jin, \& S. Lin (Eds.), Advances in computer science, intelligent system and environment (pp. 467-472). Berlin: Springer. doi: 10.1007/978-3-642-23777-5_76

Lomax, K. S. (1954). Business failures. Another example of the analysis of failure data. Journal of the American Statistical Association, 49(268), 847-852. doi: 10.1080/01621459.1954.10501239

Madi, M. T., \& Raqab, M. Z. (2004). Bayesian prediction of temperature records using the Pareto model. Environmetrics, 15(7), 701-710. doi:

10.1002/env.661

Okasha, H. M. (2014). E-Bayesian estimation for the Lomax distribution based on Type-II censored data. Journal of the Egyptian Mathematical Society, 22(3), 489-495. doi: 10.1016/j.joems.2013.12.009

Park, S., \& Balakrishnan, N. (2009). On simple calculation of the Fisher information in hybrid censoring schemes. Statistics \& Probability Letters, 79(10), 1311-1319. doi: 10.1016/j.spl.2009.02.004

Parsian, A., \& Kirmani, S. N. U. A. (2002). Estimation under LINEX loss function. In A. Ullah, A. T. K. Wan, \& A. Chaturvedi (Eds.), Handbook of applied econometrics and statistical inference (pp. 53-76). New York: Marcel Dekker.

Prakash, G. (2014). Right censored Bayes estimator for Lomax model. Statistics Research Letters, 3(1), 23-28. 


\section{GYAN PRAKASH}

Prakash, G. (2015). A comparative study based on Bayes estimation under progressively censored Rayleigh data. Journal of Statistics Applications \& Probability, 4(1), 23-30.

Prakash, G. (2017). Some Bayes estimators for Pareto Type-II progressive censored data. Electronic Journal of Applied Statistical Analysis, 10(1), 257-270. doi: 10.1285/i20705948v10n1p257

Prakash, G., \& Singh, D. C. (2013). Bayes prediction intervals for the Pareto model. Journal of Probability \& Statistical Science, 11(1), 109-122.

Singh, B., Gupta, P. K., \& Sharma, V. K. (2014). On Type-II hybrid censored Lindley distribution. Statistics Research Letters, 3, 58-62.

Singh, D. C., Prakash, G., \& Singh, P. (2007). Shrinkage testimators for the shape parameter of Pareto distribution using LINEX loss function.

Communications in Statistics - Theory and Methods, 36(4), 741-753. doi:

10.1080/03610920601033694

Singh, P. K., Singh, S. K., \& Singh, U. (2008). Bayes estimator of inverse Gaussian parameters under general Entropy loss function using Lindley's approximation. Communications in Statistics - Simulation and Computation, 37(9), 1750-1762. doi: 10.1080/03610910701884054

Steindle, J (1965). Random processes and the growth of firms, a study of the Pareto law. New York: Heffner. 